\title{
UNA AVENTURA EDUCATIVA: "EL USO DEL LIBRO DE TEXTO HACIA EL SIGLO XXI"
}

Isabel Gallardo

Resumen: Los libros de texto son una ayuda para los profesores de secundaria del país, pero muchas veces no son bien utilizados, se les considera como el compedio de todos los conocimientos. Por eso, los libros de la Serie "Hacia el siglo XXI", no han sido bien recibidos por los docentes del país, pues los obliga a ser creativos y a poner más de sí, para sacarles provecho. En este artículo, mediante un ejemplo, se desarrolla una lección en la que se usa tanto la información del libro de texto, como la creatividad de los estudiantes y la del profesor. Con el fin de sacar mayor provecho de la información que proporciona el libro de texto y de la construcción significativa del conocimiento por parte de los estudiantes.

\section{Definición de libro de texto}

¿Qué es un libro de texto? Esta pregunta es la que debe iniciar cualquier exposición que pretende hablar del uso de estos libros en el aula. Definiciones hay muchas, tantas como autores que tratan sobre el tema, por eso se escogieron tres de ellas, que se complementan una a la otra y que proporcionan un panorama más amplio para responder la pregunta inicial.

Este se puede definir como:

"Un medio de comunicación verbal y gráfico ... (donde) se da la materialización de un área del curriculum" (Mejía Botero, 1992, citado por Rojas, Marta, 1997. P. 120).

También se puede agregar que es el texto que:

"Ofrece algún desarrollo sistemático de contenidos, para la adquisición de conocimientos" (Vargas, 1995. Pp. 51, 52).

Ambas definiciones se ocupan de un aspecto importante de resaltar que es ese que se refiere al conocimiento. Se dice en una que presenta "un área de conocimiento", y la otra que ofrece "algún desarrollo sistemático de contenidos", las dos presentan con claridad la idea que en estos libros no aparece todo el conocimiento que un profesor deba tener para ofrecer a los estudiantes $\mathrm{o}$, todo lo que los alumnos necesitan para aprender. Los libros de texto presentan un fragmento seleccionado de algunos conocimientos que se han de aprender y además, 
desde una perspectiva de mundo propia, ya sea del autor del libro o de la editorial que lo produce. Es un conocimiento fragmentado, con una lectura específica de ese conocimiento y, desde un punto de vista ideológico determinado. En los libros de texto no está todo lo que se ha de enseñar, ni tampoco se encuentran las soluciones a todas las dudas de profesores y estudiantes.

Por eso, es importante añadir a las dos definiciones anteriores una más que amplía el panorama, esta propone que el libro de texto:

"Es una unidad portadora de sentidos explícitos e implícitos, así se establece una relación entre el texto, la corriente pedagógica y la sensibilidad social que intenta favorecer" (M. Rojas, 1997. P. 121).

Esta última refuerza lo dicho anteriormente y además, lo inserta dentro de una corriente pedagógica específica y una realidad social.

La elección de un libro de texto, no puede ser arbitraria, ni guiada por el colorido de su presentación o las ofertas tentadoras de las editoriales; sino más bien apoyada en la corriente pedagógica e ideológica, no solo la emanada por los entes reguladores de la educación, sino también por la que el profesor desarrolla. Tampoco se puede dejar de lado la población estudiantil que usará el texto, pues así como se presenta la visión de mundo en el libro, así se irá formando la del estudiante.

Ello enfrenta al docente al reto de escoger un texto que llene las expectativas pedagógicas y la visión de mundo, tanto suyas como la de sus estudiantes, además de sus expectativas en cuanto al contenido.

Esta última reflexión lleva a revisar cinco elementos importantes que caracterizan a los libros de texto, de acuerdo con la visión de Luis Peña (1991). En primer lugar y como ya se señaló, los libros de texto agrupan conocimientos; por eso el profesor debe revisarlos y decidir cuáles conocimientos son los que necesita que tenga el texto para cumplir con su labor en el aula. Y no solo los conocimientos sino el enfoque y el desarrollo que necesita de estos contenidos.

Otra característica, es que cada uno de los libros de texto ofrece un enfoque pedagógico específico, por lo que hay que examinar el enfoque pedagógico que maneja el profesor y el que aparece en el libro, pues ambos deben coincidir. Si el profesor se interesa en la corriente del aprendizaje significativo y el libro es más bien conductista, este podrá perderlo o desviarlo de su camino pedagógico.

Como tercera característica se encuentra que todo libro de texto, al igual que todo discurso que produce la sociedad, presenta una visión de mundo y sociedad específica y particular, generalmente adaptadas a la que tengan los escritores y los editores que pueden ser incluso, ajenas a la realidad social en que se va a utilizar el texto, pues no es lo mismo un texto preparado bajo las políticas emanadas de España o Colombia, a las emanadas de Costa Rica. Cada quien observa la realidad bajo la visión de mundo en la cual se ha desarrollado, y un editor o un escritor de textos se encontrarán inmersos en su mundo, que no necesariamente se parece o coincide con el del lugar donde posteriormente, se utilice el libro escrito y editado por esas personas. Por eso, la visión de mundo y de sociedad que tenga un libro de texto, debe adaptarse al profesor, a sus estudiantes y sobre todo, a la realidad en la cual viven y se desarrolla ese aprendizaje particular.

Como cuarta característica, se puede señalar que los textos presentan una síntesis selectiva de conocimientos. En este sentido, como se afirmó anteriormente, ningún texto presentará todos los contenidos, sino solo una selección de ellos, selección hecha por el autor o la editorial, la mayoría de las veces sin contar con la ayuda o sugerencias de docentes en ejercicio o de los mismos estudiantes. Porque aunque el texto abarque todos los contenidos que aparecen en el Plan de Estudios, la visión con que se presentan estos contenidos, el tratamiento que se les dé, la lectura que se haga, estará mediatizada, elegida y procesada desde la lente de ese 
escritor o editor que lanza el libro. Por lo tanto, los contenidos y el conocimiento que los textos presentan estará presentado bajo una óptica particular que a su vez se encuentra influida por la visión de mundo de sus autores, de la que se habló antes.

Finalmente, estos libros presentan una perspectiva particular de la realidad, y el profesor deberá revisar si esa perspectiva de realidad corresponde, o por lo menos se asemeja, a la realidad donde le corresponde enseñar para evitar una distorsión del mundo en que se desarrolla el estudiante.

Se puede concluir, entonces, que los libros de texto son una ayuda para el profesor, una guía que le permite darle variedad a la clase y un instrumento en el que se pueden realizar prácticas o ejercicios. Pero, no quiere decir que sean un sustituto del profesor, ni un compendio donde se desarrollan todos los contenidos de un año escolar, son, como ya se dijo una ayuda, no se los puede ver como la panacea que resolverá todas y cada una de las clases de un docente durante el año lectivo.

\section{La serie "Hacia el Siglo XXI"}

Antes de 1967, los libros de texto para la educación secundaria, que se encontraban en las librerías del país eran escasos, y provenían de otros países, especialmente Argentina y España. No fue sino hasta que con la creación de la Editorial Fernández Arce en Costa Rica, que escriben y venden libros de texto, sobre todo de la materia de Español. Más adelante aparecieron los libros elaborados por el Programa ODECA-ROCAP, que dotaron, por primera vez de libros a todas las escuelas del país, estos se utilizaron en la década de 1968 a 1978.

En 1985, gracias a una ayuda de la A.I.D., se elaboró la serie de textos "Hacia la Luz", para primero y segundo ciclo que se distribuyó en las escuelas públicas del país. Al dejar de reeditarse esta serie, y ante la necesidad de muchos profesores por contar con nuevos libros de texto, el mercado nacional se abrió a las editoriales privadas y extranjeras, que ofrecieron libros novedosos y agradables estéticamente.

Ante este panorama, durante la gestión de Eduardo Doryan como Ministro de Educación Pública (1994-1998), se creó una nueva serie de libros de texto, esta vez para primero, segundo y tercer ciclo, llamada "Hacia el Siglo XXI", libros que de acuerdo con palabras del Ministro, citado por Ivannia Varela "responden a los programas de estudio y son representativos de la época actual" (Varela, 1997. P. 6A).

Francisco Gutiérrez, un crítico sobre esta Serie, comenta:

“...los nuevos libros de texto, de la Serie "Hacia el Siglo XXI", tienen que responder de manera coherente a lo establecido en la norma educativa, por cuanto constituyen una estrategia concreta para abordar la calidad de la educación" (Gutiérrez, 1995. P. 15A

Este fue el objetivo que se propusieron los diferentes autores de la serie, la mayoría de ellos experimentados profesores universitarios, inmersos en la realidad costarricense y concientes de las necesidades de la educación del país; quisieron incluir, no solo los conocimientos, sino una forma significativa de aprender, donde la construcción del aprendizaje aparecía como un punto más, así como la creatividad, tanto de docentes como de estudiantes, con estas innovaciones se quería mejorar la calidad de la educación costarricense, como lo estaban pidiendo tantas voces.

Para los autores de la Serie, así como para los representantes del Ministerio de Educación Pública y algunos docentes, este conjunto de textos se adecuaban a las necesidades de la educación de la época actual (finales del siglo XX y principios del XXI). Aunque no faltaron detractores y críticos, quienes presentaron objeciones hacia estos libros.

Esta serie está integrada por un conjunto denominado "Complejo Didáctico", que incluye el libro de texto, un cuaderno de actividades (solo para primaria) y una guía para el docente. 
Como se dijo antes, desde el inicio de la gestión de Eduardo Doryan como Ministro de Educación Pública, el solo planteamiento de crear estos textos, atrajo la animosidad de varios sectores; en primer lugar de las editoriales privadas que tenían años de publicar libros de textos, quienes consideraron esta serie como una competencia "desleal", que podría acabar con sus Editoriales (1997, Bermúdez) y por otro lado el sector de los docentes.

Sobre este último punto, se comprobó en una encuesta informal, hecha en el segundo semestre del año 2000, a profesores de Español de Secundaria, del área de Desamparados, donde se observó que aunque la mayoría de los encuestados manifestó que conocía la serie de los nuevos libros de texto (un $75 \%$ ), ese mismo porcentaje, explicó que no los usa en sus clases ni se lo piden a sus estudiantes.

Las causas de este rechazo son variadas; algunos argumentaban que los contenidos que aparecen en los textos son insuficientes, otros explicaban que estos contenidos eran de regular calidad y en algunos casos confusos, y la mayoría opinaban que los contenidos de la serie "Hacia el Siglo XXI" no abarcan todos los contenidos que propone el Ministerio de Educación en sus planes de estudio.

Además, agregaban que el índice que acompaña a los libros es poco claro, que los contenidos no tienen una distribución organizada a lo largo del texto, que presentan poca práctica gramatical y que los temas se desarrollan poco.

Si estos textos se estudian desde una perspectiva objetiva, no contaminada por una visión política o por un temor a un nuevo tipo de discurso y metodología, sino situándose desde una perspectiva de aprendizaje significativo, se podrá observar que presenta aspectos no solo novedosos sino útiles para la enseñanza y el aprendizaje de la materia de Español, como se podrá observar más adelante, en el ejemplo metodológico de esta exposición.

\section{Ventajas de la serie "Hacia el Siglo XXI"}

Dentro de las ventajas de esta serie de libros, en el campo del Español, se pueden enumerar rápidamente seis aspectos importantes.

En primer lugar, a pesar de la insistencia de los profesores de todo el país, por afirmar, una y otra vez, que los libros no poseen todos los contenidos que el Plan de Estudio de Español propone, esto no es así. Los textos si abarcan todos los objetivos y contenidos que requiere el Plan de Estudio, aún aquellos que tradicionalmente han sido dejados de lado por los docentes en la enseñanza, como la expresión escrita o la expresión oral, la primera de ellas tiene un amplio desarrollo, que no se había visto en otros libros de texto usados en Costa Rica. Esto se pudo comprobar en el minucioso trabajo final del curso de la Escuela de Formación Docente, Seminario en la Enseñanza del Castellano y la Literatura, elaborado por la estudiante Gabriela Amador. En él se constató como cada uno de los objetivos y contenidos propuestos por el plan del Ministerio de Educación Pública, están desarrollados.

Un segundo aspecto, que aportan estos libros, que además es innovador, son las actividades propuestas para realizar con los estudiantes. Estas se encuentran relacionadas con el mundo de los estudiantes, son fáciles de planear y de llevar a cabo y logran promover una clase más dinámica y participativa. Estas actividades además, vienen señaladas con el dibujo de un búho, por ejemplo, cuando se estudia el teatro se le indica al usuario del libro:

"Organice, piense y lleve a cabo una dramatización informal. Como ejemplo se va a planear una. Para tener ideas, lea primero este texto" (Rojas, 1997. P. 194)

Y a continuación de la sugerencia, aparece una lectura que aporta ideas para realizar lo sugerido. 


\section{$02: 23 \mathrm{pm}$ Página 85}

Un tercer punto que también se puede destacar, es la capacidad de los autores para acercar algunos de los temas a la realidad costarricense, inclinándose por esas teorías de las que ya Paulo Freire hablaba, de fomentar el interés por lo propio de la región. Además, este acercamiento se hace evitando los estereotipos clásicos que aparecen en otros libros de texto, como ya lo señaló Mirta González Suárez (1990) en su libro sobre el sexismo en los libros de textos que se usaban tradicionalmente en las escuelas del país.
La serie estudiada pretende mostrar una realidad costarricense más actual y con la diversidad cultural que en ella se encuentra. Esta observación se puede ver reflejada, sobre todo, en las ilustraciones que acompañan al libro, como la que aparece en la página 147, del libro de sétimo año, en el tema 13 "Discutir no es pelear" (Figura 1) donde se pueden observar representantes de las diferentes etnias que pueblan el país, o en el de noveno año, en el tema 23 "El informe de investigación", por citar dos ejemplos.

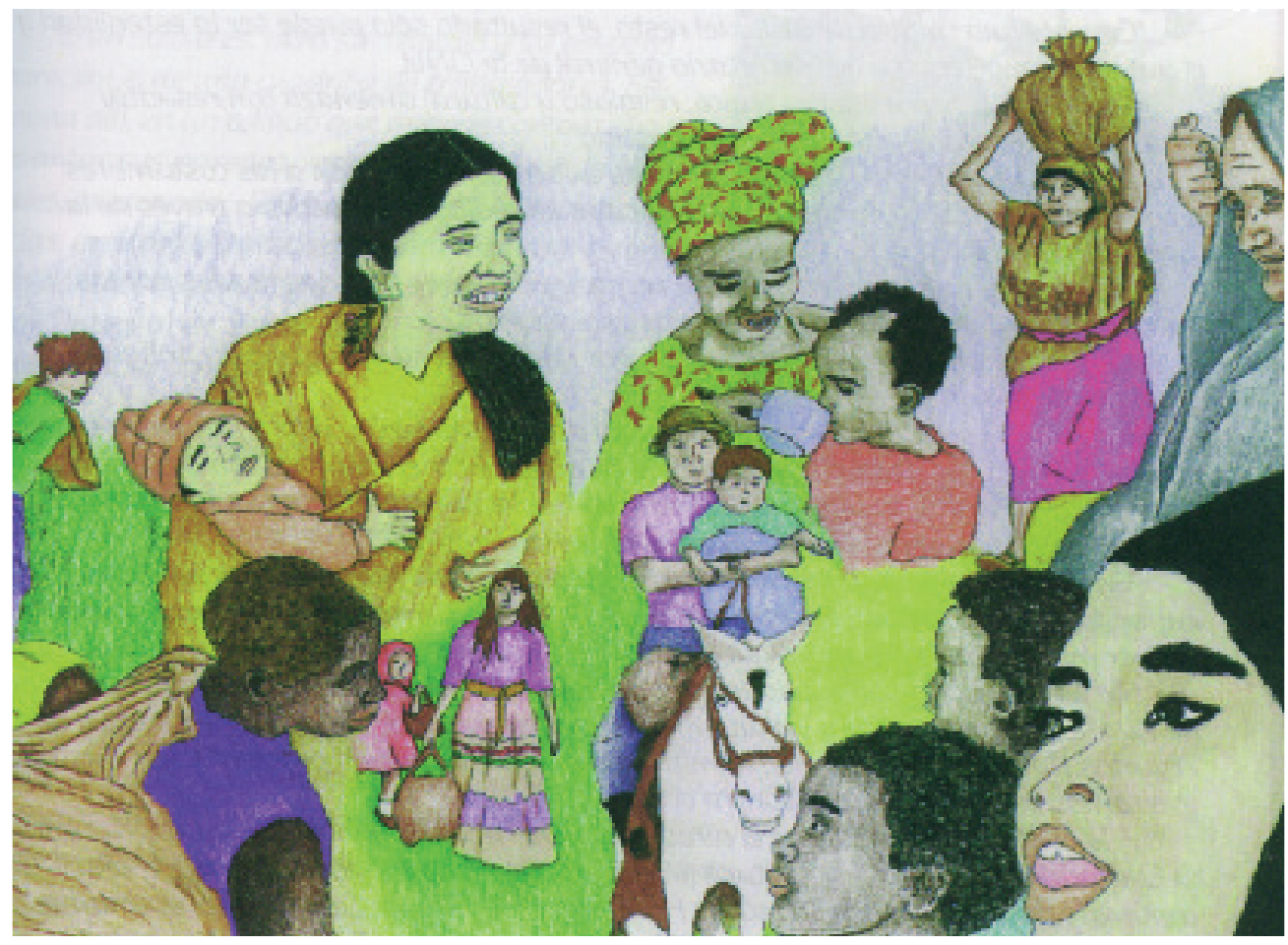

En cuarto lugar, se puede destacar el punto de vista novedoso sobre los temas tratados. Punto de vista basado en investigaciones actuales, tanto en teoría literaria, gramática, redacción como en pedagogía, que los acercan más a este mundo tan cambiante en que viven actualmente los estudiantes de los colegios costarricenses. Ejemplo de lo anterior se encuentra ya sea en las lecturas que se proponen de los diferentes textos literarios, como en la forma de estudiar la gramática, desde una perspectiva transformacional. 
En quinto lugar, lo que podría parecer una desventaja es en realidad, una ventaja para el docente y el alumno, no está dado todo el desarrollo de un contenido, como tradicionalmente lo hacen otros textos que se encuentran en el mercado nacional. Es una ventaja porque le abre posibilidades al profesor para que junto con los alumnos, presenten otras lecturas del tema y vayan más allá de lo escrito en los libros de texto, porque la serie proporciona nuevos caminos hacia donde se puede dirigir el aprendizaje del tema, basado en los contenidos ahí desarrollados. Permite, esta táctica, activar la creatividad y el pensamiento de los estudiantes y profesores que en los últimos tiempos, por causa de los medios de comunicación de masa, entre otras cosas, se encuentran como adormecidos e inactivos.

Finalmente, aunque se podrían enumerar otros aspectos positivos de esta serie, le permite al usuario investigar sobre el tema y no quedarse con la única lectura que da el texto, yendo más allá y abriendo otras puertas que no se habían abierto por miedo a lo nuevo o por desconocimiento de la posibilidad de hacerlo. Este aspecto, además, ayuda a cumplir con uno de los objetivos del Plan de Estudios propuesto por el Ministerio de Educación (Ministerio de Educación Pública. 1995) que es crear estudiantes críticos y pensantes, en contacto con su entorno y con el mundo que los rodea.

Por supuesto estos textos, como cualquier otro, tienen sus problemas, pero eso no los hace candidatos a ser descalificados de antemano, sin haber experimentado con ellos, arriesgándose a la aventura del aprendizaje diario que proponen.

Es posible trabajarlos en el aula, trascenderlos, transformar el conocimiento en un reto y en un juego, ir más allá de lo cotidiano y presentar la materia como un elemento más de la vida, útil y necesario, y no como a un intruso obligatorio, aburrido y lejano al mundo en que vive el estudiante.

\section{Un ejemplo de uso del libro "Hacia el Siglo XXI"}

Como se dijo en un inicio, el libro de texto no es un sustituto del profesor, ni es el material en que se debe basar toda la clase. Los muchachos siempre han mostrado desagrado cuando sus profesores les piden que saquen el libro de texto, lean cierto número de páginas y después resuelvan el cuestionario o la actividad final. Esta metodología tiene dos problemas, primero que no hay retroalimentación de parte ni del profesor, ni de los otros compañeros y segundo que se vuelve monótona, al repetirla clase tras clase.

Es por ello, que si se trabaja con un libro de texto, y en este caso el de Español $7^{\circ}$ de la serie "Hacia el Siglo XXI" es importante combinar técnicas de enseñanza, no solo para hacer dinámica y activa la clase, sino también, para lograr que el estudiante contribuya a crear su propio conocimiento.

Para ejemplificar esta propuesta, se tomará del libro de Español de sétimo año el capítulo "Ser románticos" (página 204 a 206. Ver el anexo) que trata sobre el Romanticismo y la poesía de Gustavo Adolfo Bécquer. Se puede conducir la clase de acuerdo con los siguientes pasos. Esto no quiere decir que sea la única manera de trabajar con el texto, y de acuerdo con la creatividad del profesor se puede variar, así como por el comportamiento del grupo durante la lección.

\section{Introducción}

Hay que iniciar la clase con una introducción que interese a los alumnos en el tema que se estudiará ese día.

El objetivo general de la lección será reconocer en la poesía de Gustavo Adolfo Bécquer algunas características del Romanticismo.

En esa motivación inicial, sin leer aún el libro, se le pide a los estudiantes que escriban en sus cuadernos la descripción física de la mujer o el hombre de sus sueños y al que 
quisieran amar. Una vez escritos pequeños párrafos en el cuaderno, se leen algunos ejemplos. (Tiempo 5 minutos).

\section{Desarrollo}

Con esta introducción se acerca al alumno al tema del día que es las características del Romanticismo en la poesía de Bécquer. Para ello se enlaza lo escrito en los cuadernos sobre un posible ser amado, con el ser amado que Bécquer plasma en sus Rimas.

Para eso, se lee, en voz alta, la Rima XI de Bécquer que aparece en la página 204 del libro de texto y que dice:

Yo soy ardiente, yo soy morena Yo soy el símbolo de la pasión;

De ansia de goces mi alma está llena; ¿a mi me buscas? -No es a ti; no

Mi frente es pálida; mis trenzas de oro; Puedo brindarte dichas sin fin; Yo de ternuras guardo un tesoro; ¿a mí me llamas? - No; no es a ti.

Yo soy un sueño, un imposible. Vano fantasma de niebla y luz; Soy incorpórea, soy intangible No puedo amarte. -¡Oh, ven; ven tú!

Se les pide que busquen en el diccionario las palabras "incorpórea" e "intangible".

Después, mediante preguntas a los estudiantes, con la finalidad de que vayan comprendiendo la Rima, se les pide, que oralmente describan físicamente a la mujer de la primera estrofa, así como su forma de amar. Se escriben las características en la pizarra. Se hace lo mismo con la mujer de la segunda estrofa, y también se anotan sus características.

Una vez descritas las dos primeras mujeres se preguntan las diferencias entre ambas.

Luego, se vuelve a la tercera mujer del poema, se formulan las mismas preguntas y se anotan sus características. Se comparan ahora, la primera y segunda mujer con la tercera. Se les pregunta a cuál de las tres prefiere el yo lírico y cómo será el estado de ánimo de ese yo lírico para preferir a la tercera mujer, en lugar de las dos primeras. (Tiempo 10 minutos).

\section{Género y Movimiento}

Una vez que se ha empezado a comprender el contenido de la Rima, se clasifica el texto en el género y el movimiento al que pertenece.

Para eso, se lee en voz alta la página 205 del libro de texto. Una vez leído, mediante una lluvia de ideas se pregunta qué es género y qué es movimiento, y se escribe en la pizarra lo que los estudiantes van diciendo. Una vez vistos ambos términos, se clasifica la Rima leída en el género y en el movimiento a que pertenece. Hay que recordar que los estudiantes ya han visto, desde sexto grado, y quizá antes, lo que es lírica, por lo que se pueden completar las ideas del texto, con los conocimientos que ellos poseen sobre el tema.

Finalmente, volviendo a referirse a lo leído en el libro de texto se discute porqué los conceptos antes definidos son convenciones.

Se les puede decir a los alumnos que copien en sus cuadernos los conceptos que se generaron con la lluvia de ideas. Hay que recordar que al ir generando ellos mismos los conceptos, basados en lo que han leído, va a ser más claro lo que copien y el tema va a quedar más firme y claro. (Tiempo 10 minutos).

\section{Características del Romanticismo}

Una vez ubicado el poema en el movimiento, la clase se dedica a buscar las características del movimiento Romántico, pero, solo aquellas que es posible ver reflejadas en la Rima XI. 
La primera característica que se busca es el rompimiento de las convenciones.

Para ello, se les pregunta a los estudiantes, cuantos hablantes aparecen en el poema y cuáles son estos hablantes, se recuerda a las diferentes mujeres que se describieron al inicio de la clase y las respuestas que el yo lírico le dio a cada una.

Para llegar a descubrir la característica de ruptura de las convenciones, se les pide que recuerden otros poemas estudiados en clase o, si no se acuerdan se puede leer cualquiera de los poemas que aparece en el mismo libro de texto, páginas más adelante.

Se pregunta cuántos hablantes aparecen en ese nuevo poema recordado o leído. Se compara el número de hablantes con el de la Rima XI, y se acota que en los poemas casi siempre aparece un solo hablante o yo lírico. Esto presenta una característica de Bécquer, que es cambiar lo establecido, la norma usada hasta ese entonces. Se les dice que redacten esa característica en el cuaderno.

La segunda característica romántica que se buscará es el amor que nunca se puede alcanzar.

Para eso se vuelve a las mujeres del poema. Se pregunta de nuevo porqué la tercera es diferente, porqué se cree que es la preferida del yo lírico. Se solicita que describan como puede ser un amor de ese tipo. Después deben redactar esa característica.

Una vez encontradas y redactadas las dos características se les pide que lean, en voz baja, la página 206 del libro y que comparen las características redactadas por ellos con las que allí aparecen. Esta lectura les confirmará que la que construyeron estaba bien hecha y afianzará, aún más, lo aprendido. (Tiempo 20 minutos).

\section{Actividad Complementaria o de cierre}

Una vez analizado el contenido del poema se puede hacer una actividad complementaria que produzca un complemento al aprendizaje hasta ahí obtenido.
En pequeños grupos, de no más de cuatro personas, se les dice que con recortes o dibujos, representen en una mitad del papel, como se imaginan que es la mujer ideal que aparece en el poema de Bécquer, y en el otro extremo del papel que representen al hombre o a la mujer ideal que describió, al inicio de la clase, uno de los integrantes del grupo.

Luego se exponen los dibujos ante la clase y se les pregunta en qué se diferencian el amor romántico del amor actual, y si les hubiera gustado vivir en la época Romántica o no y porqué. Así podrán iniciar la comprensión de las diferencias de vida en cada época, y como el mundo no ha sido siempre igual a como ellos lo conocen ahora. (Tiempo 30 minutos).

\section{Materiales}

Para llevar a cabo esta clase, que puede tomar dos lecciones, los materiales que se requieren no son muchos, y todos ellos son fáciles de conseguir. Son:

- $\quad$ Libro de texto, sétimo año de la Serie "Hacia el Siglo XXI"

- Papel periódico o de construcción para hacer el dibujo

- Revistas o periódicos

- $\quad$ Lápices o marcadores de colores

- $\quad$ Tijeras y goma.

\section{Observaciones finales}

El acto de la enseñanza y del aprendizaje, es un acto que requiere de paciencia y esfuerzo, tanto del docente como del estudiante. Ambos tienen que poner de su parte para lograr un aprendizaje significativo, que más que una cantidad de datos retenidos en la memoria, sea un ejercicio de investigación, observación y construcción; no es tan importante proporcionarle al estudiante diez o trece características del Romanticismo, que se

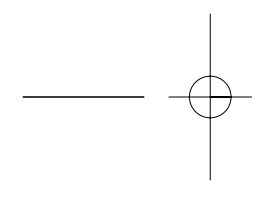


aprenderá de memoria para un examen y que luego olvidará, como enseñarlo, mediante las preguntas y la observación a que ellos mismos descubran esas características en un texto escrito, y que así desarrollen su capacidad de investigación.

Hay que recordar que esta Rima XI, no va a ser la única Rima de Bécquer que se estudiará en clase, hay más y de esas otras, se pueden ir deduciendo otras características del Romanticismo, esto ayudará a los estudiantes no solo a ser más observadores sino más críticos con el material que se les presente.

No se quieren estudiantes que repitan, que memoricen sin entender, sino muchachos que sean capaces de observar el mundo y sacar consecuencias de él, criticarlo y soñar con mejorarlo. Esa es la idea, no solo de este modelo de clase aquí presentado sino de los libros de texto de la serie "Hacia el Siglo XXI", siempre y cuando se usen con imaginación y creatividad.

El libro de texto, tanto estos de la serie estudiada, como cualquier otro, deben usarse como un complemento de la enseñanza, como un arma más para que estudiante conozca y no como la única forma de enseñar y la única materia que se les proporciona. Si se usa de esta forma, como el compendio de todos los conocimientos, el profesor estará restringiéndole el mundo al alumno y a sí mismo, además de promover clases muy pasivas y memorísticas.

Ya decía J. Novak (1982) que la memoria es un factor de aprendizaje de corto plazo, es muy fácil olvidar lo que se memoriza, y memorizar es lo que en muchos casos se está haciendo en el proceso de enseñanza aprendizaje. Pero, actualmente, no se busca estudiantes que repitan lo que dice el profesor o el libro de texto, se buscan estudiantes críticos, pensantes, investigadores y concientes de su entorno. Para esto se deben tomar en cuenta todos los materiales que se tengan a mano, incluyendo el libro de texto, usándolo de manera que el muchacho aprenda a leer su mundo en ellos y a prepararse para vivir en ese mundo actual, tan complejo y difícil que le tocó vivir.

\section{Referencias bibliográficas}

Amador S., Gabriela. "Aporte de los libros de texto como material de apoyo para enseñar literatura en sétimo año de secundaria" Trabajo presentado para el curso FD-0550 Seminario en la Enseñanza del Castellano y la Literatura. Universidad de Costa Rica. 1999.

Bermúdez, Manuel. "Libros para alquilar y manzana de la discordia" En: Semanario Universidad. San Pedro, 21 de febrero. 1997.

Fernández Lobo, Mario. "Análisis de los textos escolares costarricenses y las normas de calidad propuestas por el Consejo de Educación" En: Comunicación y Cultura. San José, I semestre. 1996.

Garro M., Cinthya. "Libros de Texto "Serie: Hacia el Siglo XXI". Trabajo presentado para el curso FD-0550 Seminario en la Enseñanza del Castellano y la Literatura. Universidad de Costa Rica. 2000.

González Súarez, Mirta. El sexismo en la educación. San Pedro, Editorial Universidad de Costa Rica. 1990.

Gutiérrez, Francisco. "Los libros de texto" En: La Nación. San José, 7 de julio. 1995.

Mejía, William. ¿Sabe usted escoger sus textos escolares? Bogotá, Centro Norma de Apoyo al Docente. 1992.

Ministerio de Educación Pública. Programas de Estudio Tercer Ciclo, Español. San José, Departamento de Publicaciones MEP. 1995. 
Novak, Joseph D. Teoría y práctica de la educación. Madrid, Alianza Editorial. 1982.

Peña, Luis. "El libro de texto como problema de la calidad educativa" En: La calidad del libro de texto. Bogotá, Editorial Plaza Impresoras. 1991.

Ramírez Escobar, José Alonso. "Textos didácticos: un acercamiento semiótico" En: Revista Educación. San Pedro. Volumen 21, No 2. 1997.

Rojas M., Juan Luis. "Comparación, análisis de algunos libros de texto que hay en el mercado nacional" Trabajo presentado para el curso FD-0550 Seminario en la Enseñanza del Castellano y la Literatura. Universidad de Costa Rica. 1998.

Rojas, Marta. "El libro de texto: instrumento para la innovación educativa" En: Revista Educación. San Pedro, Volumen 22, $\mathrm{N}^{\circ} 1.1997$.

Rojas, Marta y otros. Español $7^{\circ}$ : texto. San Pedro, Editorial Universidad de Costa Rica. 1996.

Rojas Marta y otros. Guía Didáctica, San Pedro, Editorial Universidad de Costa Rica. 1996.

Varela, Ivannia. "Retornan los libros de texto oficiales" En: La Nación. San José, 28 de enero. 1996. 
ANEXO N 1

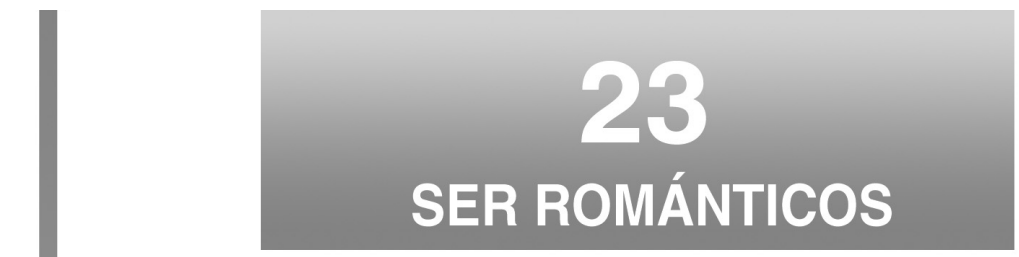

XI

-yo soy ardiente, yo soy morena, yo soy el simbolo de la pasion: de ansia de goces mi alma estállena.

¿A mi me buscas? -No es a ti; no.

- Mi frente es pálida; mis trenzas, de oro; puedo brindarte dichas sin fin: yo de ternura guardo un tesoro. ¿A mi me llamas? -No; no es a ti.

- Yo soy un sueño, un imposible.

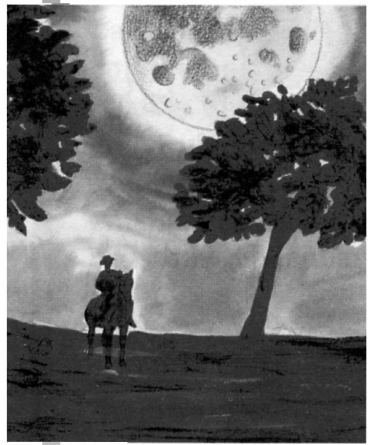
vano fantasma de niebla y luz; soy incorporea, soy intangible; no puedo amarte. -iOh, ven; ven tu!

Gustavo Adolfo Bécquer RJMAS (1868)

¿Qué le parece esta pequeña muestra de romanticismo? ¿Qué cree usted que significa este último término? En esta parte se va a estudiar qué es el romanticismo, gracias al análisis de un texto de narrativa y uno de lírica que representan este movimiento literario. Además, se va a elaborar un esquema para el análisis de textos narrativos y líricos, luego de haber estudiado varios de ellos. 


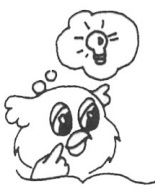

He aquí, pues, un nuevo tema dentro de los estudios literarios: el de los movimientos literarios. El romanticismo es uno de esos movimientos, y es el que interesa en sétimo año. Pero, ¿qué es, en general, un movimiento literario? ¿Tiene usted alguna idea al respecto?

Buena parte de los textos literarios pertenecen no solamente a un género literario, sino también a un movimiento literario. Por ejemplo, se dice de la anterior poesía de Bécquer que es del género lírico y del movimiento romántico.

Un movimiento se parece a un género en el sentido de que es, básicamente, una convención o acuerdo. Quienes participan de un mismo movimiento, acuerdan utilizar el lenguaje literario de ciertas maneras. Este acuerdo sirve, posteriormente, para guiar al lector, quien sabe que, puesto a leer un texto propio de un movimiento dado, habrá de encontrar ciertas formas de hacer literatura.

La diferencia con respecto a los géneros literarios se da porque los movimientos ocurren en periodos históricos más o menos definidos, mientras que los géneros suelen tener una vigencia más prolongada. Es decir, los movimientos literarios ocurren en ciertos lugares durante ciertas épocas: los géneros en cambio, aunque también son históricos, a menudo se mantienen. Así, por ejemplo, el romanticismo es un movimiento literario originario de Europa, desde donde llega a otros lugares de Occidente, y que tiene lugar durante el siglo XIX, sobre todo en su primera mitad.

Interesa saber ahora cuáles son las características predominantes del movimiento romántico, es decir, cuáles son los principales acuerdos o convenciones que rigieron históricamente el movimiento y que hoy funcionan como guías para la lectura. Además de ser una convención lingüística, un movimiento literario crea toda una forma de relacionarse con el mundo. Los movimientos literarios interpretan o representan la realidad de ciertas formas. Ser romántico, por ejemplo, significa leer la realidad de la forma en que los románticos acordaron hacerlo.

Un movimiento literario crea una forma histórica de hacer literatura y de representarse la realidad. 
ANEXO No 3

Vuelva ahora al "Poema XI", de Bécquer. En él, hallará algunas de las características del movimiento romántico. ¿Cuáles son? Comparta sus ideas con el resto del grupo. Luego compárelas con las siguientes características:

1. Este poema presenta la particularidad de que en él aparecen varios hablantes, en lugar de uno solo. Esto nos demuestra que, como se había dicho antes, los géneros literarios no tienen reglas fijas: aunque lo usual en la lírica es que solo haya un hablante, aquí se tiene una excepción. Al romántico le gusta innovar, no se atiene a todas las reglas.

2. Ahora bien, cada estrofa presenta un diálogo en el que el primer hablante se caracteriza a sí mismo y luego cede la palabra a un segundo hablante, que es el mismo en las tres estrofas y que se puede considerar el yo lírico. ¿Cree usted que el primer hablante es el mismo en las tres estrofas, o se dan tres hablantes distintos?

3. ¿Cómo describiría usted al primer hablante de la primera estrofa y al primer hablante de la segunda estrofa?

Representan (1) la pasión

(2) la ternura
Tienen en común el ser reales y estar en disposición de amar.

4. ¿Cómo describiría usted al primer hablante de la tercera estrofa? ¿Qué puede querer decir el hecho de que es a ella a quien prefiere el yo lírico?

El ideal amoroso romántico es un imposible: el romántico ama y sabe que no puede alcanzar al ser amado, precisamente porque se enamora de imposibles. 